\title{
EPILEPTIC SEIZURE TRIGGERS IN CHILDREN FROM ROMANIA
}

\author{
Anca Adriana Arbune, Oana Tarta Arsene², Lacramioara Brinduse, Dana Craiu² \\ ${ }^{1}$ Neurology Department, University Emergency Hospital Bucharest - Neurology Clinic, \\ "Carol Davila" University of Medicineand Pharmacy, Bucharest \\ 2Department 6 Clinical Neurosciences, Pediatric Neurology Clinic, "Prof. Dr. Alexandru Obregia" \\ Psychiatry Clinical Hospital, "Carol Davila” University of Medicineand Pharmacy, Bucharest \\ ${ }^{3}$ National Public Health Institute, "Carol Davila" University of Medicineand Pharmacy, Bucharest
}

\begin{abstract}
Objectives. The identification of the types, characteristics and correlations of epileptic seizure triggers in children with epilepsy from Romania.

Material and methods. Transversal observational study on 278 children with epilepsy using the questionnaire method, containing questions regarding seizure characteristics, demographical information and medical history. Results. The lot characteristics were average age 9.74 years; $57.2 \%$ males; $19.4 \%$ positive epilepsy familiy history; 48.2\% late psychomotor development; $54 \%$ deficitary neuropsychological development; $25.2 \%$ treatment resistant epilepsies; $59.7 \%$ of children had focal epileptic seizures, $35.3 \%$ had generalized. Average number of triggering factors was 3 and $20.1 \%$ of children had no seizure trigger. The most frequently reported factors were: sleep deprivation $39.57 \%$, anxiety $23 \%$, sleep disturbances $22.66 \%$, fever $20.50 \%$, anger $19 \%$, light stimulation $17.27 \%$, crying $16.19 \%$, interrupting antiepileptic medication administration $14.39 \%$, watching too much TV $14.39 \%$, physical fatigue $12.59 \%$. The number of seizure triggers can be mathematically modelled according to age and treatment resistance. Unresponsiveness to treatment of the epilepsy has a few predictors: timing of the seizure ocurrence, imaging alterations, late psychomotor development, IQ, the number of seizure triggering factors.

Conclusions. There was at least one seizure trigger identified in $79.9 \%$ of children with epilepsy. The most frequent reported trigger was sleep deprivation. The number of seizure triggers can be estimated through mathematical modelling. Treatment resistance of epielptic seizures has more predicting factors.
\end{abstract}

Keywords: epilepsy, seizure triggers, children

\section{INTRODUCTION}

Epilepsy is the most common neurological affliction in the world, with a major social impact. The World Health Organisation estimates that there are 2.4 milion new cases per year diagnosed, half of which are children or adolescents (WHO, 2012). Most people with epilepsy could lead a normal life with proper treatment, that would efficiently control seizures.

Many epileptic patients recognise certain relationships between their seizures and certain factors, more or less specific. Nonspecific,general factors (such as fever, stress, fatigue, etc.) are not related to the type of epilepsy. Specific factors, such as light or noise, usually identify a reflex epilepsy.

The currently best known triggers in the medical literature are visual stimuli (intermittent light, pat- terns, video or computer games) (Fisher, Harding et al. 2005), audio stimuli (music, sounds, noise) (Lin, Wang et al. 2003), tactile or somatosensitive stimuli (Hsieh, Chiou et al. 2011), startle (Yang, Liu et al. 2010), reading (Striano and Striano 2011), writing (Chifari, Piazzini et al. 2004), praxia (Glenn, Carrazana et al. 2012), eating (Rosenzweig and Manford 2008), hot water (Yalcin, Toydemir et al. 2006), calculus (Marino, Lanzafame et al. 2009), thinking of a certain subject (Striano, Minetti et al. 2010; Nevler and Gandelman-Marton 2012), board games (Lee, Yoo et al. 2012).

The study of epileptic seizure triggers in children is important and is justified by the limitations of current drug therapy, that does not achieve the optimal seizure control. As a consequence, the prevention of seizures in treatment resistant epilepsies 
is possible only by making lifestyle changes and avoiding seizure triggers.

The objectives of our study were the identification of seizure triggers in children with epilepsy in Romania and the evaluation of their corelations, in the view of improving the current management protocols of epilepsy.

\section{MATERIAL AND METHODS}

The current study is an observational transversal study regarding children with epilepsy who were admitted to the Neuropediatric Clinic of "Prof. Dr. Al. Obregia" Psychiatry Clinical Hospital in Bucharest between 01.01.2013 and 31.12.2013. Ages varied between 1 and 18 years. Babies under 1 year old were excluded due to the fact that epilepsy diagnosis at this age is often uncertain and frequently confused with isolated febrile seizures.

Demographic data, medical history and results of the psychological evaluation were collected from 357 medical files of eligible patients, whose parents were approached to take part in the study. Only 278 parents accepted to participate, answering our custom made questionnaire, 44 refused and 35 could not be contacted.

The questionnaire contained a list of 40 items, out of which parents identified the factors they observed to trigger epileptic seizures in their children. The psychological evaluation used the Wechsler Intelligence Scale for Children (WISC-IV) (Wechsler, 2003). The results of the test differentiate between average intelligence (IQ 90-110), under average (IQ 80-89), borderline (IQ 70-79) and mental retard $(\mathrm{IQ} \leq 69)$.

The obtained data was statistically analyzed using Microsoft Excel XLSTAT Pack and SPSS 15. We described the variables, made Pearson and Spearman bivariate correlations with significance level $\mathrm{p}<0.05$ and multivariate analysis.

The study was approved by the Hospital Ethics Committee.

\section{RESULTS}

The age of the patients varied between 2 and 18 years old. The average age was 9.47 years with the standard deviation of 4.645 years and the median age was 9.81 years.

The demographic characteristics of the children included in the study are found in Table 1.

The epilepsy diagnoses were very diverse and the epilepsy classification system changed often in the last decades, thus we preferred to take note of
TABLE 1. Demographic characteristics of children with epilepsy in Romania

\begin{tabular}{|l|c|c|c|c|}
\hline & Number & Percent & P & Cl 95\% \\
\hline Gender & & & & \\
M & 159 & $57.2 \%$ & & \\
F & 119 & $42.8 \%$ & 0.016 & $0.51-0.63$ \\
\hline Living area & & & & \\
Urban & 147 & $52.9 \%$ & & \\
Rural & 131 & $47.1 \%$ & 0.337 & $0.46-0.58$ \\
\hline Child Rank & & & & \\
I & 151 & $54.3 \%$ & 0.150 & $0.48-0.60$ \\
II & 84 & $30.2 \%$ & $<0.001$ & $0.24-0.35$ \\
$\geq$ III & 43 & $15.5 \%$ & $<0.001$ & $0.11-0.19$ \\
\hline IQ & & & & \\
Low & 150 & $54 \%$ & 0.187 & $0.48-0.59$ \\
Normal & 127 & $45.6 \%$ & 0.150 & $0.39-0.52$ \\
Superior & 1 & $0.4 \%$ & & \\
\hline \multicolumn{5}{|l}{} \\
\hline
\end{tabular}

the types of seizures: $59.7 \%(\mathrm{~N}=166)$ focal, $35.3 \%$ $(\mathrm{N}=98)$ generalized and 5\% $(\mathrm{N}=14)$ unclassified, both focal and generalized.

The type of epileptic seizure correlated with the weight at birth category $(p=0.039)$. Children under $2,500 \mathrm{~g}$ at birth, as well as those over $4,000 \mathrm{~g}$, had in a significantly greater number focal seizures. The greatest percent of children with normal weight at birth was observed in children with generalized seizures $(87.75 \%$ vs. $74.7 \%$ focal seizures and $78.57 \%$ unclassified seizures).

The timing of seizure occurrence was noted, $56.1 \%(\mathrm{~N}=156)$ of the children having their seizures predominantly when awake and approx. $25 \%$ $(\mathrm{N}=68)$ having seizures regardless of the moment of the day. A correlation with the number of reported seizure triggers was observed $(p=0.044)$. The children with seizures anytime in the day have a high number of seizure triggers (average: $2.74 \pm$ 1.663), probably due to excessive reporting, the parents interpreting as seizure trigger any incidental factor, without any real correlation. Children with seizures when falling asleep or waking up have an average of seizure triggers of $3.15 \pm 2.544$, the highest average with the highest standard deviation. Children with seizures while awake have an average of $2.81 \pm 2.094$ potential triggers, while children with seizures during sleep have the least average in the lot, 1.66 seizure triggers, as expected taking into account the difficulty in relating anything with the seizures.

Another correlation was observed, between timing of the seizures and treatment resistance $(p<0.001)$, children with seizures anytime in the day being less responsive to antiepileptic drugs in terms of seizure control, while children with seizures when waking up or falling asleep having better outcome. 
TABLE 2. Similar studies on epileptic seizure triggers

\begin{tabular}{|c|c|c|}
\hline Study & Country & \begin{tabular}{|c}
$\%$ of patients who \\
identified epileptic \\
seizure triggers
\end{tabular} \\
\hline $\begin{array}{l}\text { (Konishi, Naganuma et al. } \\
\text { 1992) }\end{array}$ & Japan & $51.5 \%$ \\
\hline $\begin{array}{l}\text { (Nakken, Solaas et al. } \\
\text { 2005) }\end{array}$ & Norway & $53 \%$ \\
\hline $\begin{array}{l}\text { (Chowdhury, Hasan et al. } \\
\text { 2014) }\end{array}$ & India & $62 \%$ \\
\hline (Frucht, Quigg et al. 2000) & USA & $62 \%$ \\
\hline $\begin{array}{l}\text { (Sperling, Schilling et al. } \\
\text { 2008) }\end{array}$ & USA & $65 \%$ \\
\hline $\begin{array}{l}\text { (Spatt, Langbauer et al. } \\
\text { 1998) }\end{array}$ & Austria & $71 \%$ \\
\hline $\begin{array}{l}\text { (Tan, Wilder-Smith et al. } \\
\text { 2005) }\end{array}$ & Singapore & $75 \%$ \\
\hline $\begin{array}{l}\text { (Lunardi Mdos, Sukys- } \\
\text { Claudino et al. 2011) }\end{array}$ & Brazil & $84.5 \%$ \\
\hline (Spector, Cull et al. 2000) & Great Britain & $90 \%$ \\
\hline $\begin{array}{l}\text { (da Silva Sousa, Lin et al. } \\
\text { 2005) }\end{array}$ & Brazil & $92 \%$ \\
\hline (Ferlisi and Shorvon 2014) & Italy & $97 \%$ \\
\hline Present study (2014) & Romania & $79.9 \%$ \\
\hline
\end{tabular}

Treatment resistance was identified in $25.2 \%$ $(\mathrm{N}=70)$ of the cases. Among the resulting correlations, worth mentioning are the positive associations with late psychomotor development $(\mathrm{p}<0.001)$, the presence of imaging abnormalities $(p=0.012)$ and low IQ $(\mathrm{p}<0.001)$, mainly because a higher seizure frequency profoundly affects the child's development and intelect, the presence of a distinctive lesion indicating a clear source of a more difficult to control seizure onset zone. As expected, the number of seizure triggers was significantly higher $(\mathrm{p}=0.029)$ in the case of children with treatment resistant seizures in comparison with responsive children (averages 3.11 vs 2.48).

The potential predictors of treatment resistance were associated in blocks in a multiple hierarchical regression. In accordance with the level of statistical correlation with treatment resistance the following variables were introduced: psychomotor development $(p<0.001)$, seizure timing $(p<0.001)$, IQ $(\mathrm{p}<0.001)$, imaging abnormalities $(\mathrm{p}=0.012)$ and the number of reported seizure triggers $(\mathrm{p}=0.022)$. Other studies also identified some of these factors as predictors: late psychomotor development and imaging abnormalities (Gururaj, Sztriha et al. 2006; Seker Yilmaz, Okuyaz et al. 2013). The final model indicated that the psychomotor development is a negative predictor $(B=-0.07)$, so as timming of the seizures $(B=-0.11)$ and IQ $(B=-0.17)$, while imaging abnormalities $(B=0.05)$ and the number of reported seizure triggers $(B=0.02)$ were found as positive predictors.
The number of seizure triggers identified by parents varied between 0 and 10, with median 3 and average 2.64. A percentage of $20.1 \%(\mathrm{~N}=56)$ of children had no observed seizure trigger, the rest of $79.9 \%$ having reported at least one. We identified only one similar study in the available literature, with a lot made up exclusively of children (Konishi, Naganuma et al. 1992). Out of all the children with epilepsy, 50\% ( $\mathrm{N}=139)$ had between 1 and 3 potential seizure triggers and $29.9 \%(\mathrm{~N}=83)$ had more than 3 .

The first important correlation observed was between the number of triggers and the age $(p<0.001)$, the number of observed factors increasing proportionally with age, most likely due to the increase in observational and expressive capacity, but also because parents could easier identify seizures as the epilepsy evolved or the number of recognized seizures increased. On the other hand, adolescents frequently have more risk behaviours, being predisposed to more frequent seizures caused by alcohol, drugs, caffeine, lack of sleep and stress.

The correlation with treatment resistance $(p=0.022)$ adds another problem to the unresponsiveness to antiepileptic drugs: sensitivity towards a larger number of triggers means a greater risk of having a seizure, thus a greater number of seizures, entering a vicious circle. At the same time, treatment resistance means an increased number of seizures, thus a bigger risk of falsely identifying potential triggers.

We used the multiple hierarchical regression in which we introduced age as the first independent variable and treatment rezistance as the second. Age significantly contributes $(\mathrm{p}<0.001)$ to the prediction of the number of triggers with $4.9 \%$. If we add antiepileptic drugs resistance, the variance in the number of seizure triggers increseas to $6 \%$, with a significance of $p=0.041$. Thus we can calculate the number of potentional seizure triggering factors in a child with epilepsy using the formulas:

No. seizure triggers $=1.673+$ Age $* 0.099$

No. seizure triggers $=1.572+$ Age $* 0.095+$ Treatment resistence $* 0.555$

Figure 1 illustrates the frequency of all the reported seizure triggers by the parents of children with epilepsy included and willing to take part in the study.

Numerous correlations between different seizure triggering factors and other data (clinical, anamnestic, psychological, imaging data), and also with other triggers. The results were concentrated 


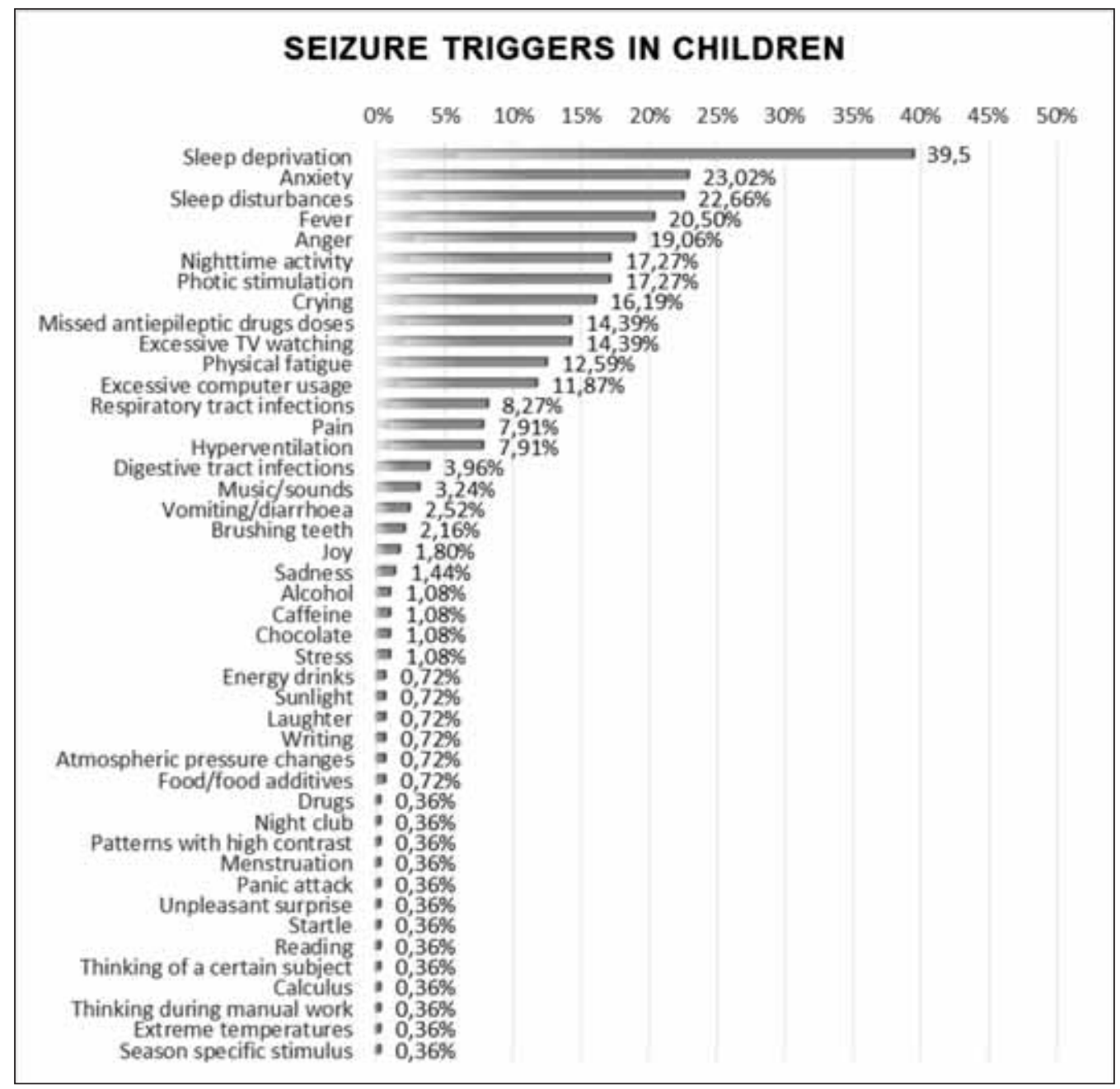

FIGURE 1. Frequencies of epileptic seizure triggers in children

in a few observations and logical schemes (Figures $2 a, b, c)$ about the modality of questioning patients about epileptic seizure triggers.

\section{DISCUSSIONS}

Among the children who took part in the study, parents identified at least one seizure trigger in $79.9 \%$ of cases, the percentage being similar to other studies, although most included adults and only one included aduls and children when questioning about seizure triggers. Seelp deprivation was also identified as a very frequent trigger, but other triggers that we asked about were not included in the questionnaires.

When a treatment resistant epilepsy is diagnosed in a child, parents and patient, when old enough, should be questioned on the reaction to fury or intense anger, reaction to fatigue, to music or sounds. The first correlation can be explained by the fact that children with treatment unresponsiveness live more often the frustration of lack of results in spite of optimal conduct and efficient drugs prescribed. These negative feelings can increase and cause a negative output on life and loss of tolerance to other negative situations, such moments becoming seizure triggers. Another correlation to take into account is that between anger/fury and fatigue $(p=0.003)$, explained by their interdependance, biochemical stress in the body predisposing to irritabillity, the seizures being ieasier provoked by negative emotional states.

A surprising correlation found was between anxiety as triggering factor and type of seizure $(p=0.047)$, children with focal seizures having anxiety significantly more frequent as trigger. This correlation can occur possibly due to more frequent involvement of mesial structures in focal epilepsy, superimposing with the networks responsible for fear and anxiety perception. Moreover, caffeine and chocolate consumption provoked significantly more frequent focal seizures.

Another interesting relationship was found between anxiety and excessive computer use as sei- 


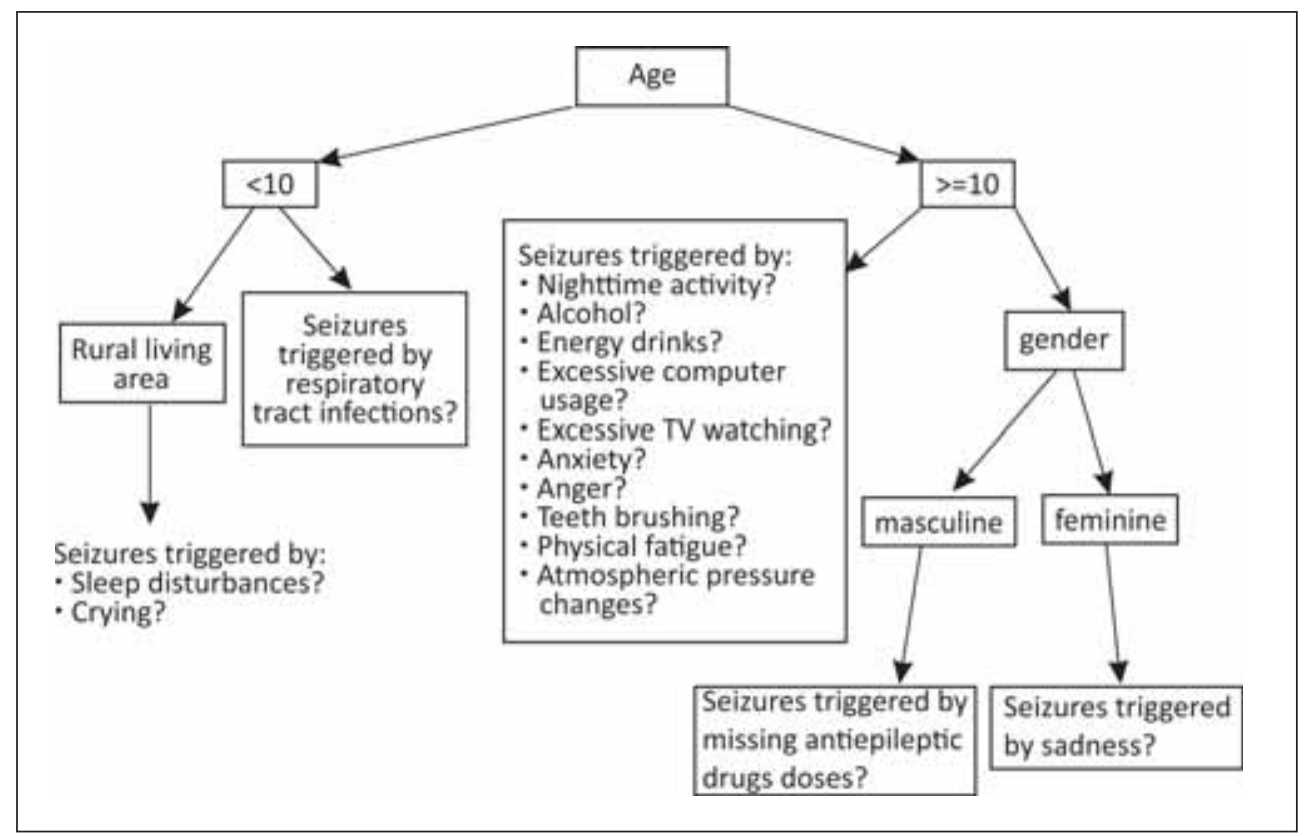

a)

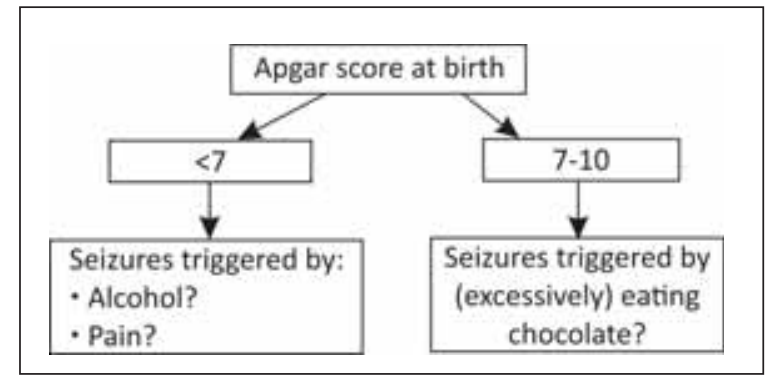

b)

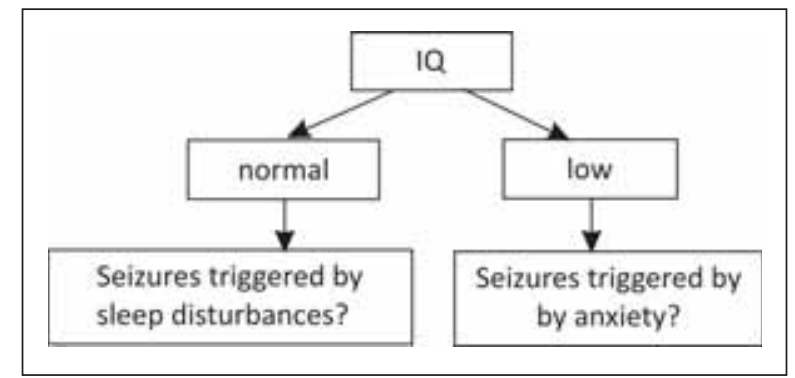

c)

FIGURE 2. Logic schemes of structured anamnesis on seizure triggers according to: a) age, gender and living area; b) Apgar score at birth; c) IQ.

zure triggers $(\mathrm{p}=0.043)$, children having seizures after spending too much time in front of a computer having significantly less frequent seizures when feeling anxious and viceversa. A possible explanation would be that violent games (first person shooter, horror, thriller), often played by children these days, by sending visual impulses first through the amygdala, then to the optical perception areas, hyperstimulate the amygdala due to the threatening visual content. The vegetative answer to fear is the increase of heart beats, respiratory frequency and body temperature, leading to the association with hyperventilation and extreme temperatures as seizure triggers $(p<0.001$ and $r=0.006)$ in these children. In time, the fear response attenuates and tolerance appears, anxiety having not enough effect to cause seizures.

Small weight at birth of a child with epilepsy should lead to questioning about the susceptibility to high contrast patterns or light, surprising touch.
Premature birth predisposes children with epilepsy to seizure triggers such as excessive crying and sleep disturbances, which are metabolic stressful situations that easily cause an imbalance in susceptible neuronal zones.

If the epileptic child has a positive family history, epilepsies with genetic components should be investigated, such as epilepsies which have pain, reading, mathematical calculus and music as seizure triggers.

When the child had febrile convulsions in his/ her personal history, stroboscopic lights and extreme happiness can be among their seizure triggers, also suggesting the implication of the amygdala in the epileptogenesis.

Head trauma was correlated with many seizure triggers: panic attacks, unpleasant surprise, voluntary lack of sleep, reading, eating and certain foods, all of these indicating the corresponding affected cortical areas by the trauma or preexistent lesions aggravated by the trauma. 


\section{CONCLUSIONS}

Epileptic seizure triggers in children represent a practical subject of interest regarding optimal seizure control, especially due to the negative effects of frequent seizures on a developing brain, during the most important period in learning and acquiring basic skills. Avoiding seizure triggers can be of great help in epilepsy management, especially when treatment resistance occurrs.

Taking into account that $79.9 \%$ of children with epilepsy included in the study identified at least one seizure trigger, extensive research of triggers represents an important subject for future studies.

The results of the present study were synthetized in a few probability schemes for different sei-

\section{REFERENCES}

1. Beck S., Wojdyla D., Say L., Betran A.P., Merialdi M., Requejo J.H., Rubens C., Menon R. Van Look P.F. (2010). "The worldwide incidence of preterm birth: a systematic review of maternal mortality and morbidity". Bull of the World Health Organ 88:31-38.

2. Chifari R., Piazzini A., et al. (2004). "Reflex writing seizures in two siblings with juvenile myoclonic epilepsy." Acta Neurol Scand 109(3): 232-235.

3. Ferlisi M., Shorvon S. (2014). "Seizure precipitants (triggering factors) in patients with epilepsy." Epilepsy Behav 33: 101-105.

4. Glenn M., Carrazana E.J., et al. (2012). "Late-onset, praxis-induced myoclonic epilepsy." Epileptic Disord 14(2): 167-171.

5. Gururaj A., Sztriha L., et al. (2006). "Clinical predictors of intractable childhood epilepsy." J Psychosom Res 61(3): 343-347.

6. Hsieh C.Y., Chiou N.J., et al. (2011). "Somatosensory rub evoked reflex epilepsy of a temporal lobe origin." Neurol Sci 32(2): 297-299.

7. Konishi T., Naganuma Y., et al. (1992). "Seizure-inducing factors in the patients with childhood epilepsy." No To Hattatsu 24(3): 238-243.

8. Lee M.K., Yoo J., et al. (2012). "Reflex epilepsy induced by playing Go-stop or Baduk games." Seizure 21(10): 770-774

9. Lin K.L., Wang H.S., et al. (2003). "A young infant with musicogenic epilepsy." Pediatr Neurol 28(5): 379-381.

10. Marino S., Lanzafame P., et al. (2009). "Neurofunctional assessment in a case of calculation-induced seizures." Neurol Sci 30(6): 505-508.

11. Nevler N., Gandelman-Marton R. (2012). "Acute provoked reflex seizures induced by thinking." Neurologist 18(6): 415-417. zure triggers according to different parameters and can be seen in Figures $2 a, b, c$. These schemes can further be used to elaborate an anamnesis protocol for children with epilepsy, as well as a more comprehensive lifestyle advice sheet.

More detailed studies on a greater number of children could validate these schemes for clinical use, aiding in the identification and control of triggering factors, especially when dealing with treatment resistant epilepsies.

We consider that more studies are needed on the subject, with the possible inclusion in the questionnaire of questions regarding possible seizure inhibiting factors.

12. Rosenzweig I., Manford M. (2008). "Trouble at dinner: an unusual case of eating-induced seizures." J Neurol Neurosurg Psychiatry 79(3): 335-337.

13. Seker Yilmaz, B., Okuyaz C., et al. (2013). "Predictors of intractable childhood epilepsy." Pediatr Neurol 48(1): 52-55.

14. Striano P., Minetti C., et al. (2010). "Lesional reflex epilepsy associated with the thought of food." Neurology 75(3): 288-289; author reply 289 .

15. Striano P., Striano S. (2011). "Reading epilepsy and its variants: a model for system epilepsy." Epilepsy Behav 20(3): 591.

16. Yalcin A.D., Toydemir H.E., et al. (2006). "Hot water epilepsy: clinical and electroencephalographic features of 25 cases." Epilepsy Behav 9(1): 89-94.

17. Yang Z., Liu X., et al. (2010). "Clinical and electrophysiological characteristics of startle epilepsy in childhood." Clin Neurophysiol 121(5): 658-664.

18. Wechsler D., Kaplan E., Fein D., Kramer J., Morris R., Delis D., Maelender A. (2003). Wechsler intelligence scale for children: Fourth edition (WISC-IV) [Assessment instrument]. San Antonio, TX; Pearson.

19. World Health Organization (WHO). (2012). "Epilepsy". WHO Factsheet, N999. Accessed Jan. 2, 2016 Available at: www.who.int/ mediacentre/factsheets/fs999/en/index.html 